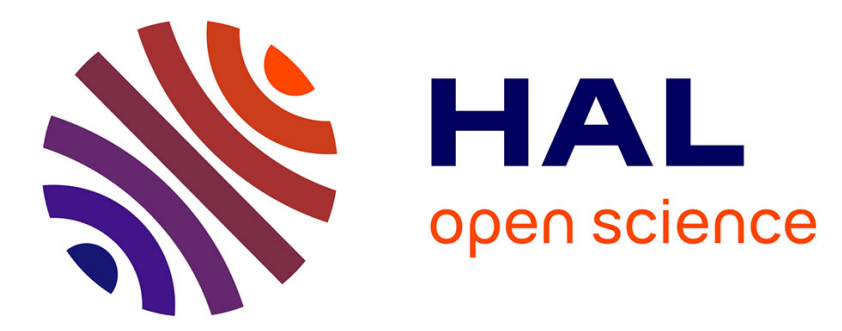

\title{
Conformational plasticity of hydrogen bonded bis-urea supramolecular polymers
}

P. Brocorens, M. Linares, Carine Guyard-Duhayon, R. Guillot, B. Andrioletti, D. Suhr, B. Isare, R. Lazzaroni, Laurent Bouteiller

\section{- To cite this version:}

P. Brocorens, M. Linares, Carine Guyard-Duhayon, R. Guillot, B. Andrioletti, et al.. Conformational plasticity of hydrogen bonded bis-urea supramolecular polymers. Journal of Physical Chemistry B, 2013, 117 (17), pp.5379-5386. 10.1021/jp401915y · hal-00993522

\section{HAL Id: hal-00993522 \\ https://hal.science/hal-00993522}

Submitted on 27 Aug 2020

HAL is a multi-disciplinary open access archive for the deposit and dissemination of scientific research documents, whether they are published or not. The documents may come from teaching and research institutions in France or abroad, or from public or private research centers.
L'archive ouverte pluridisciplinaire HAL, est destinée au dépôt et à la diffusion de documents scientifiques de niveau recherche, publiés ou non, émanant des établissements d'enseignement et de recherche français ou étrangers, des laboratoires publics ou privés. 


\section{Conformational Plasticity of Hydrogen Bonded Bis-urea}

\section{Supramolecular Polymers}

Patrick Brocorens, ${ }^{\circ}$ Mathieu Linares, ${ }^{,}$Carine Guyard-Duhayon, ${ }^{\circ}$ Régis Guillot, ${ }^{\circ}$ Bruno Andrioletti, Dominique Suhr,' Benjamin Isare, Roberto Lazzaroni, 'a and Laurent Bouteiller"

¿Service de Chimie des Matériaux Nouveaux, Université de Mons /Materia Nova, Place du Parc, 20, B7000 Mons, Belgium

${ }^{\circ}$ Department of Physics, Chemistry and Biology (IFM), Linköping University, SE-58183 Linköping, Sweden

'Laboratoire de Chimie de Coordination du CNRS, BP 44099, 205 route de Narbonne, 31077 Toulouse Cedex 4, France

‘nstitut de Chimie Moléculaire et des Matériaux d'Orsay, UMR CNRS 8182, Bât. 420 Université ParisSud, 91405 Orsay cedex, France

•Université Claude Bernard-Lyon 1, ICBMS-UMR 5246, 43 Boulevard du 11 Novembre 1918, F-69622 Villeurbanne cedex, France

' UPMC Univ Paris 06, UMR 7610, Chimie des Polymères, F-75005 Paris, France, and CNRS, UMR 7610, Chimie des Polymères, F-75005 Paris, France

$\begin{array}{lllll}\text { AUTHOR } & \text { EMAIL } & \text { ADDRESS } & \text { laurent.bouteiller@upmc.fr } \quad+33 \quad(0) 144276179\end{array}$ roberto.lazzaroni@umons.ac.be+32 (0)65373860

KEYWORDS - Self-assembly - Molecular simulation - Filament - Dynamic 
ABSTRACT. We report a detailed structural investigation of supramolecular polymers formed by hydrogen bonded self-assembly of bis-urea monomers. The careful exploration of the energy landscape by Molecular Mechanics/Molecular Dynamics (MM/MD) simulations has allowed to identify three distinct self-assembled structures of similar stabilities. These structures have been compared to X-ray crystal data. We observe that a slight change in the molecular structure can favor a particular structure over the others. Detailed analysis shows that hydrogen bonds stabilize all three structures to a similar extent. Therefore, it is the interactions among the lateral substituents, and with the filament environment, that are the decisive factors in the competition between the possible self-assembled structures. This study constitutes a clear reminder that the conformation of a supramolecular polymer is a sensitive function of the molecular structure and may significantly differ from the solid-state conformation of a model compound.

\section{Introduction}

Supramolecular polymers are chains of small molecules held together through reversible non-covalent interactions. ${ }^{1-4}$ Compared to usual covalent polymers, the dynamic character of the interaction is responsible for the appearance of new properties, such as self-healing behavior or improved processability. Moreover, their high molar mass structure is maintained only by weak interactions, which makes them highly sensitive to small perturbations. Therefore, supramolecular polymers are particularly interesting systems enabling the quantification of subtle self-assembly features such as chiral amplification ${ }^{5}$ or solvation effects..$^{67}$

Advances in this field are directly linked to our ability to rationally improve the design of a given supramolecular polymer, which in turn requires to know its precise structure. However, this is a difficult task because of the lack of long-range order in these systems and because of their dynamic character. A popular approach to circumvent this problem is to use modern molecular simulation tools validated by experimental data. ${ }^{8-10}$ Often, a model compound similar to the supramolecular polymer is studied

experimentally, for instance by X-ray diffraction on crystals, to obtain structural information. ${ }^{11-15}$ These data can then be used to select and validate a simulation method to assess the conformation of the supramolecular polymer. However, this is still a significant challenge, because the conformation of the model compound in the crystal is not necessarily the most stable conformation of the solvated assembly, 
as solid-state packing effects distort the energy landscape. This approach therefore requires a comprehensive exploration of the energy landscape of the supramolecular assemblies to identify the most relevant conformations and arrangements.

Besides determining the most stable structures, the molecular modeling approach also allows to highlight the richness of the structural variability of a given class of polymers and can provide a description of those structures with a unique level of details. This is particularly interesting for the class of bis-urea based supramolecular polymers studied in this work. In principle, the assembly of these molecules can be favored/controlled by hydrogen-bonding between the urea groups, $\pi$-stacking between the toluene units and interactions between the hydrocarbon side groups.

In non-polar solvents (such as toluene or dodecane), 2,4-bis(2-ethylhexylureido)toluene (bis-urea 1) (Figure 1) has been shown to self-assemble by hydrogen bonding into two distinct supramolecular structures, which are stable over a wide range of concentrations and temperatures, and are in dynamic exchange with the monomer. ${ }^{16-18}$ A small angle neutron scattering (SANS) study established that both structures are long and fibrillar, the high temperature structure being thinner than the low temperature structure. Throughout this work, we focus exclusively on the high temperature structure, which has been shown by SANS to consist of single stranded filaments. Quantitative measurements of the strength of hydrogen bonds by IR spectroscopy and isothermal titration calorimetry (ITC), and comparison to data for mono-ureas revealed that both urea groups of a given monomer are hydrogen-bonded to the next monomer in a cooperative fashion, i.e. random association of urea groups can be excluded..$^{1920}$ Based on those data and on the structure of a monolayer probed by scanning tunneling microscopy (STM), ${ }^{21-23} \mathrm{a}$ schematic ladder-like supramolecular arrangement has been proposed. ${ }^{16}$ However, many degrees of freedom are present in this molecule, for instance related to the flexibility of the side groups and the relative orientation of the methyl groups on adjacent toluene units in the assemblies; as a result, considerable uncertainty remains concerning its organization in the solvated assembly.

It is thus the aim of the present study to reach a detailed structural description of the supramolecular filaments and an evaluation of their energetic stability, via a joint approach combining experimental structural studies and Molecular Mechanics/Molecular Dynamics (MM/MD) simulations of model compounds for the filaments. 
Needle-like crystals suitable for X-ray diffraction have been obtained for the closely related molecules 2 and 3 (Figure 1). On one hand, these two compounds can be used to set up and validate a simulation methodology to study assemblies of bis-urea 1 molecules. On the other hand, they allow to extend the investigation to bis-urea supramolecular polymers differing by the nature of the substituents. Finally, we considered bis-urea $\mathbf{4}$, which has a very small side group, i.e., a methyl group, and should thus reveal the most stable conformation of the bis-urea core in the absence of any steric hindrance from the substituents.

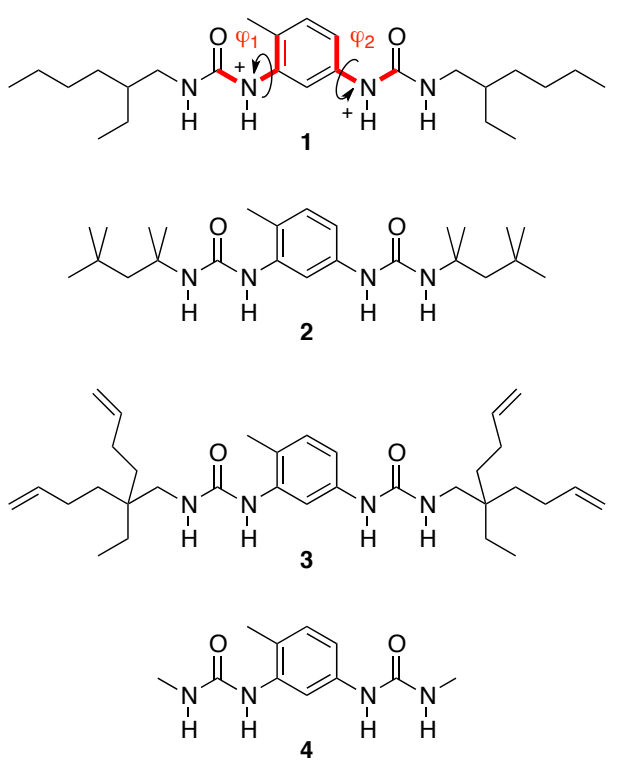

Figure 1: Structure of the bis-urea monomers examined in this study; the torsion angles $\varphi_{1}$ and $\varphi_{2}$ are noted on $\mathbf{1}$ (the arrows indicate in which direction the torsion angle is considered as positive).

\section{Methodology}

The bis-ureas 1 to 4 were modeled with the Dreiding ${ }^{24}$ force field using the Materials Studio modeling package. The validity of Dreiding for this study was evaluated on single molecules versus a quantum chemical method. Special attention was dedicated to the behavior of the torsions, which are the internal coordinates most influencing the supramolecular organization. The comparative analysis - available as Supporting Information - indicates the suitability of Dreiding, which is further reinforced by the fact that this force field reproduces correctly the experimental solid-state morphology of bis-ureas $\mathbf{2}$ and $\mathbf{3}$ (see below). 
After validating the force field, a methodology to generate filament structures as exhaustively as possible has been devised. To that aim, we performed conformational searches for a single molecule, then for dimers, and then for octamers, i.e. for assemblies of increasing size, to funnel the search towards the most stable structures having a complete hydrogen bond network. That approach was applied to the simplest compound, bis-urea 4, to assess the dominant role of the hydrogen bonds (among the other intermolecular interactions) in stabilizing molecular assemblies.

Firstly, the potential energy surface of bis-urea 4 was explored by systematically varying the orientation of the urea groups with respect to the aromatic unit $\left(\varphi_{1}\right.$ and $\varphi_{2}$ in Figure 1) in a single isolated molecule. That work also provided the stable conformations of the bis-urea core in the absence of intermolecular interactions. Then, the stable conformations obtained were used to build hydrogenbonded dimers. Among them, however, only a few have an arrangement that can be extrapolated to a fully hydrogen-bonded filament; thus only a few octamers with a periodic motif were built subsequently from these dimers.

When building the octamers, the stacked molecules were placed so that the methyl groups on adjacent toluene rings have alternating orientation, ${ }^{25}$ in order to reduce steric hindrance (see the motif of helical and straight structures in Figure 2). That strategy produces only filaments with a repetitive motif of at most two molecules. We also included the repetitive motif of six molecules that is experimentally observed in the crystal structure of bis-urea 2 (Figure 2, zigzag structure). In total, five conformations were obtained for each bis-urea compound, but only three of them keep their structure during molecular dynamics simulations. We will mainly discuss these three persistent structures, which are referred to according to the shape of their morphology: helical, straight, or zigzag. The two unstable structures are discussed in Supporting Information.

Once these stable morphologies have been obtained, the influence of the steric effects from the side groups was investigated. The three filament configurations were systematically studied for bis-ureas $\mathbf{1}$ to $\mathbf{4}$, generating 12 different structures in total. Filaments of 32 molecules were built, geometryoptimized by Molecular Mechanics and then submitted to 1500 ps Molecular Dynamics simulations. Data for analysis were collected every 3 ps after the initial 150 ps of trajectory, when most strong fluctuations have disappeared. To analyze the systems, the distribution of torsions is displayed as an 
average of 5 bins of $1^{\circ}$ each, and the evolution of the potential energy is displayed as block averages calculated on ten structures.

The MM energy minimizations were performed with a conjugate gradient algorithm until a convergence criterion of $0.001 \mathrm{kcal} / \mathrm{mol} . \AA$ ' was reached. The long-range interaction cutoff distance was set to $14 \AA$ with a spline width of $3 \AA$. The charges were assigned from the pcff force field.

The MD simulations were performed in the NVT ensemble (i.e., particle number, volume, and temperature are kept constant). The Nosé thermal bath coupling scheme ${ }^{26}$ was used to maintain the temperature at $300 \mathrm{~K}$, with a coupling constant of 0.1 . A Verlet velocity algorithm ${ }^{27}$ was used to integrate the equations of motion with a $1 \mathrm{fs}$ time step. All structures in our study were relaxed prior to MD simulations.

\section{Results}

\section{Experimental structure of filaments in the solid state}

The first clues about the organization of bis-urea $\mathbf{1}$ in filaments and the driving forces leading to them were obtained by analyzing the X-ray diffraction data of the closely related systems, bis-ureas 2 and $\mathbf{3}$. Bis-urea 3 crystallizes in a monoclinic cell $\left(a=17.566 \AA, b=23.795 \AA, c=8.651 \AA, \beta=117.40^{\circ}\right)$, forming straight filaments with a structural motif of two molecules repeating along c. A given molecule has both urea groups tilted with respect to the phenyl core by the same absolute value $\left(129.3^{\circ}\right)$ and with the same sign, but the sign alternates from one molecule to the next (see Figure 1 to localize the torsions $\varphi_{1}$ and $\varphi_{2}$ that describe the orientation of the urea groups). This type of filament morphology is referred to as straight, and is displayed in Figure 2. (Note that all morphologies represented in Figure 2 were those obtained by simulation and correspond to bis-urea 4 filaments, which are used to describe characteristics common to all four bis-urea compounds and common to both crystalline and simulation conditions. For the representation of the actual crystal structures of bis-urea 2 and $\mathbf{3}$, see Supporting Information.

Bis-urea 2 crystallizes in a monoclinic cell $\left(a=19.628 \AA, b=18.277 \AA, c=24.896 \AA, \beta=112.503^{\circ}\right)$. The structural motif has six molecules repeating along c, with kinks appearing every three molecules, thus leading to zigzagging filaments. This type of filament morphology is thus referred to as zigzag, and 
is displayed in Figure 2. (Note that in the example displayed, the zigzag shape is more visible when looking at the hydrogen bond network; see also Figure S3). Each motif is composed of two triads of molecules. In the first triad, the central molecule has both urea groups tilted by $131.8^{\circ}$. For the other two molecules, one urea is tilted by $125.7^{\circ}\left(\varphi_{2}\right)$, the other one by $137.6^{\circ}\left(\varphi_{1}\right)$. In the second triad, the torsions are similar, except that the sign is negative. As the sign changes when going from one triad to the next, the two frontier molecules are organized as in the straight motif (compare the straight motif and the two central molecules of the zigzag motif in Figure 2). In each triad, the position of the methyl group on the toluene ring of the central molecule is undefined in the crystal structure data. This orientational disorder is consistent with the fact that the orientation of the methyl group on the central molecule alternates with the methyl group of any of the two neighboring molecules, but not with the methyl group of both molecules.

In summary, these two bis-urea based monomers crystallize differently due to the nature of their side groups, but remarkably both structures in the solid state are filaments where all urea moieties are involved in hydrogen bond. As experimental evidence suggests that bis-urea 1 also forms filaments in solution (although crystal data are not available), these results point out that filament structures have a strong tendency to form for this class of supramolecular polymers, mainly driven by the formation of intermolecular hydrogen bonds. 

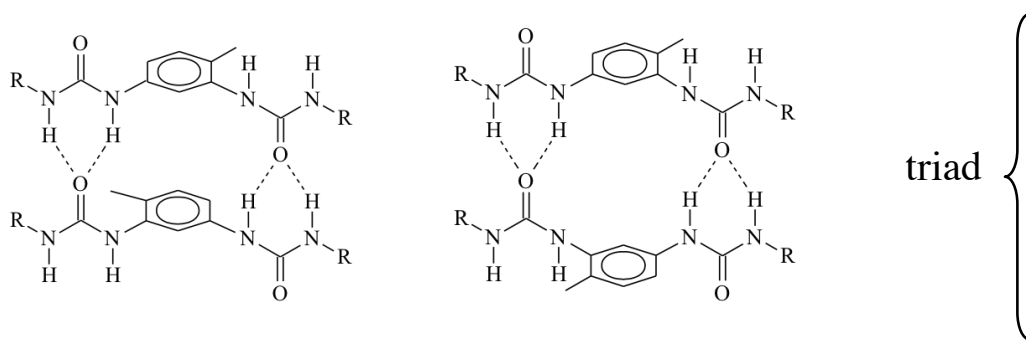

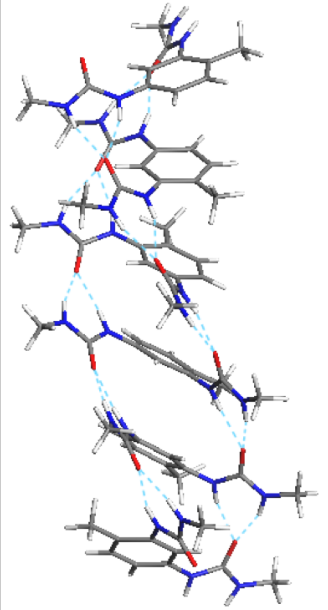

Helical

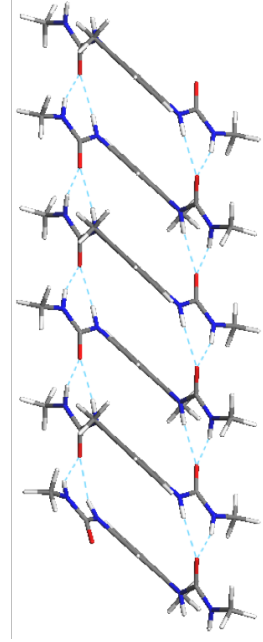

Straight

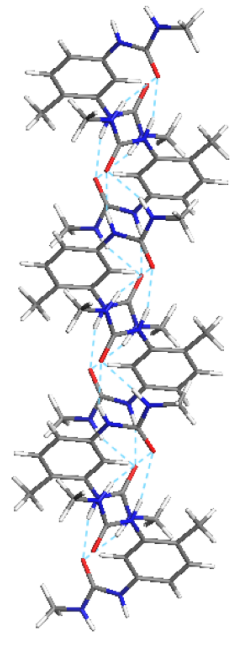

triad
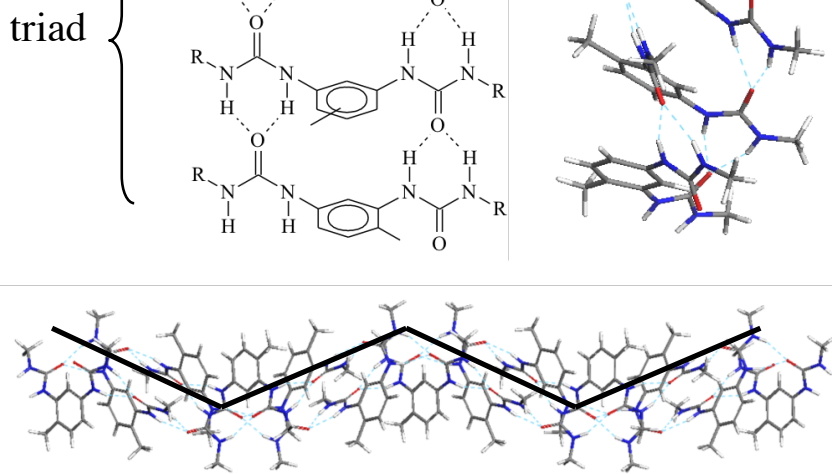

Zigzag

Figure 2: Orientation of the bis-urea molecules in the filament morphologies discussed in this study. The 3D displays correspond to bis-urea 4 , i.e. where the substituent $\mathrm{R}$ of the schematic motif is a methyl, and were obtained by a molecular mechanics simulation. For the straight filament, two views are displayed, along two perpendicular directions. 


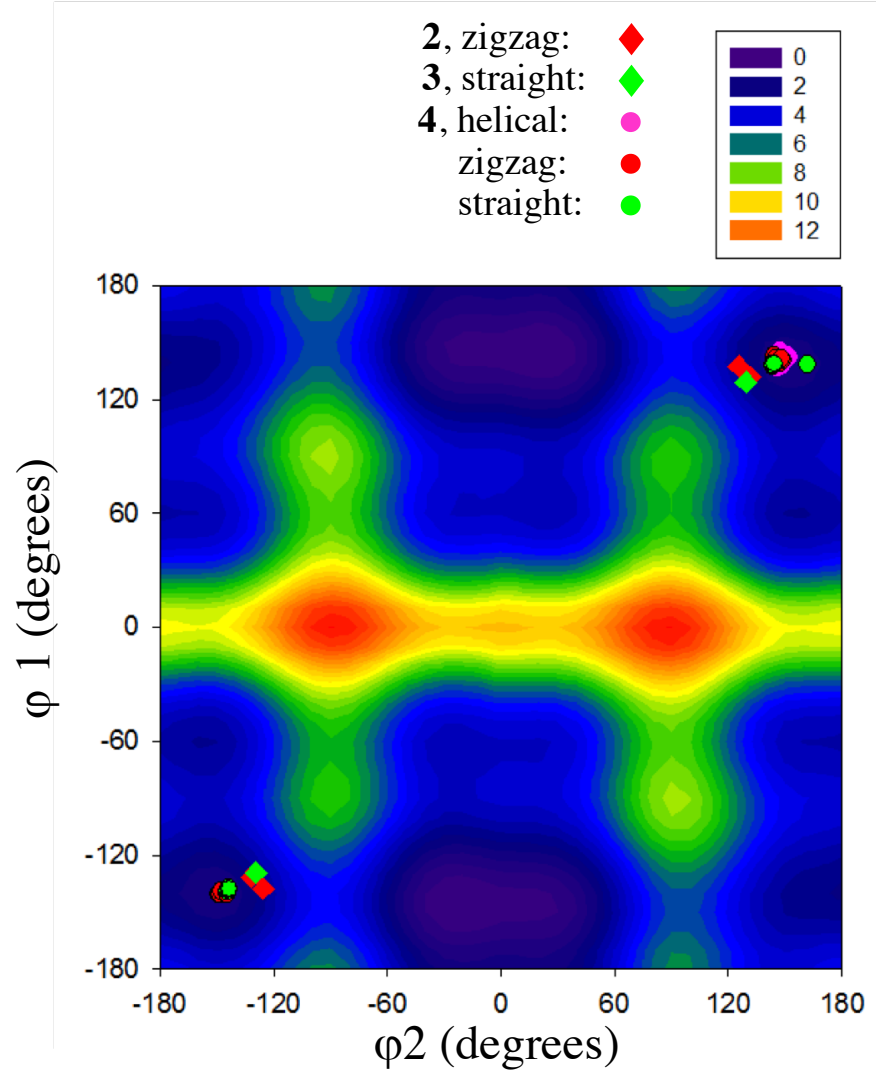

Figure 3: Map of relative potential energy $(\mathrm{kcal} / \mathrm{mol})$ of an isolated bis-urea 4 molecule built by varying $\varphi_{1}$ and $\varphi_{2}$, showing the preferential orientations of the urea moieties with respect to the core. The values corresponding to the helical, zigzag and straight conformations for a simulated single filament of bis-urea 4, and the zigzag and straight conformations for the experimental crystalline filaments of bis-ureas $\mathbf{2}$ and $\mathbf{3}$, respectively, are located on the map.

\section{Simulations of single filaments}

The potential energy map of a single bis-urea 4 molecule (Figure 3) reflects the preferential orientations of the urea moieties with respect to the core in the absence of intermolecular interactions. The potential energy is minimal for $\left|\varphi_{1}\right|=150^{\circ}$ and $\left|\varphi_{2}\right|=20^{\circ}$, but around that minimum, conformations requiring less than the thermal energy to be accessible at room temperature are found for $\varphi_{1}$ between - 
$130^{\circ}$ and $-160^{\circ}$, and between $130^{\circ}$ and $160^{\circ}$, and $\varphi_{2}$ between $-40^{\circ}$ and $40^{\circ}$; in other words, the global minimum is in a potential energy valley with a large flat bottom, where the urea groups can be almost coplanar with the ring or tilted by up to a few tens of degrees.

On this map the filament conformations determined from X-ray diffraction data or from simulation are visualized as diamonds or circles, respectively. None of them are located in the area of minimal potential energy of the monomer. This means that to form the stabilizing hydrogen-bond network, the urea moieties have to deviate from their preferential orientation in isolated molecules. It is actually in the second lowest potential energy valley, where $\left|\varphi_{1}\right|$ is about $130-150^{\circ}$ and $\left|\varphi_{2}\right|$ is about $140-170^{\circ}$, that the zigzag, straight, and helical morphologies are located.

It is remarkable to have similar torsions in these three morphologies, considering the differences between them: the size and shape of the side groups, the environment (crystal or vacuum), the morphology of the filament itself (helical, zigzagging, or straight). It is thus likely that both the intramolecular interactions associated to the orientation of the urea moieties, and the intermolecular interactions associated to hydrogen bonding synergistically stabilize the assemblies when $\left|\varphi_{1}\right|,\left|\varphi_{2}\right| \sim 125-$ $160^{\circ}$. Within that constraint, the morphology still conserves some freedom to vary and respond to packing or steric effects induced by the substituents, by tuning the frequency of the $\varphi_{1}, \varphi_{2}$ sign change along the filament. This sign alternation is seen on the energy map (Figure 3), where the zigzag and straight morphologies have two well-defined, symmetrically-positioned populations of $\varphi_{1}, \varphi_{2}$ : the sign alternates from half a motif to the next (i.e. each molecule or each triad for the straight and zigzag morphologies, respectively). Helical filaments, on the contrary, have only one population of torsion angles (all $\varphi_{1}$ and $\varphi_{2}>0$ in a M-helix or all $\varphi_{1}$ and $\varphi_{2}<0$ in a P-helix).

The relative adaptability of the filament morphologies to different conditions is further illustrated during the MD simulations performed on the unstable bis-urea 4 filaments: as mentioned in Methodology, two of the initial five filament morphologies are unstable during the simulations, and one of them evolves towards the more stable helical morphology in a few picoseconds (see Supporting Information). However, in the course of this morphology conversion, a few bis-urea molecules do not 
follow the main path and adopt conformations with both $\varphi_{1}$ and $\varphi_{2}$ close to $60^{\circ}$ (Figure 4 ). As a result the filament folds in two. What is remarkable is that only a few torsional "defects" introduced in the helical morphology are sufficient to fold the filament, while conserving the full integrity of the hydrogen bond network. This observation suggests that bis-urea filaments have the possibility to fold and adapt their morphology to changes of their environment without much energy penalty.
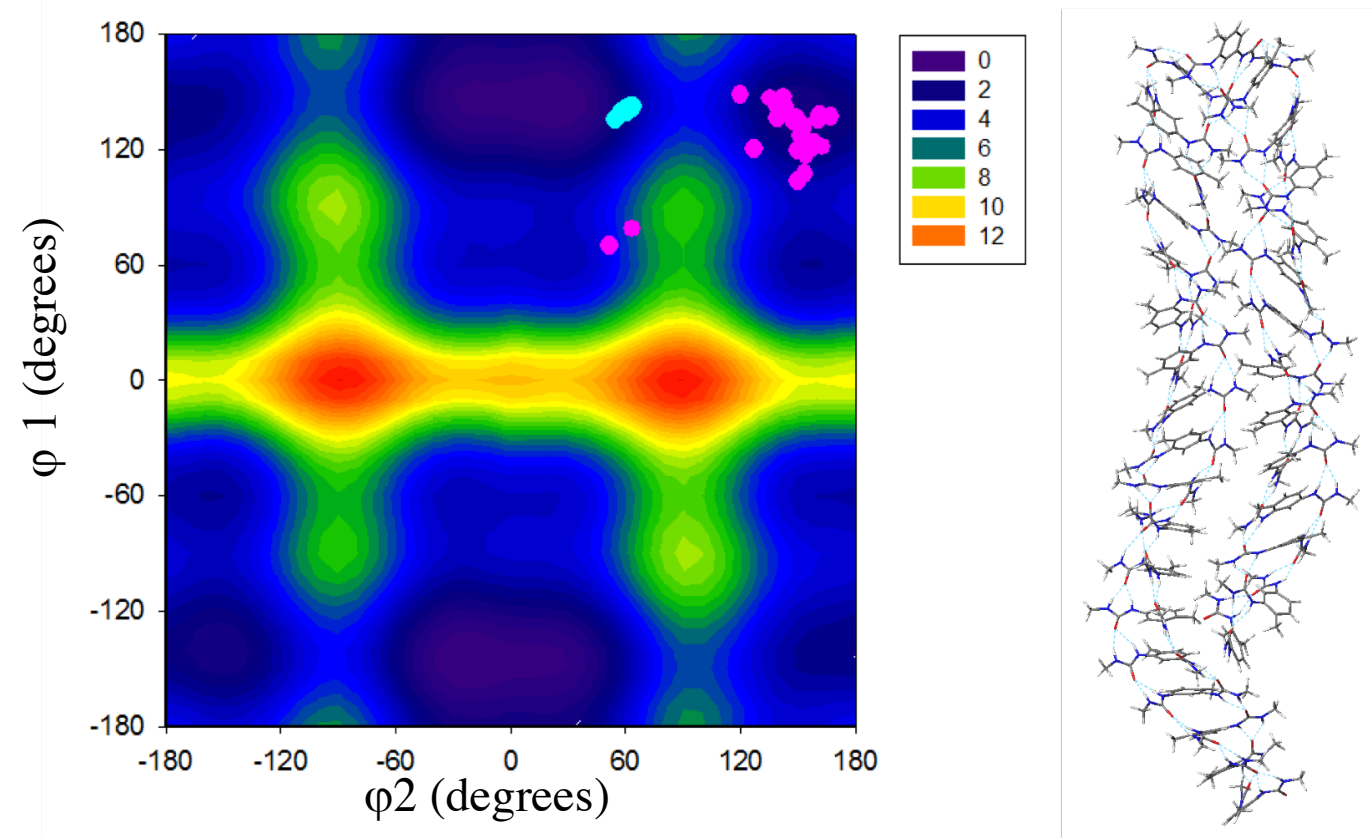

Figure 4:

Map of relative potential energy ( $\mathrm{kcal} / \mathrm{mol})$ of an isolated bis-urea 4 molecule built by varying $\varphi_{1}$ and $\varphi_{2}$.

The values for a simulated unstable filament of bis-urea 4 are located on the map before $\left({ }^{\bullet}\right)$ and after

(•) 1500 ps dynamics. The folded structure obtained after MD simulation is displayed on the right.

Figures 5 and 6 show the H-bond energy and the total potential energy, respectively, for the filaments of the four bis-urea compounds in the course of the MD simulations, which allows to estimate the relative stability of the helical, zigzag, and straight morphologies. 
As far as H-bond energy is concerned, bis-urea $\mathbf{4}$ is the system that has the strongest hydrogen-bond network, with similar strength for the three morphologies (Figure 5). This is probably due to the small size of the substituents, which limits steric hindrance. However, larger substituents do not destabilize much the H-bond network: at most $1 \mathrm{kcal} / \mathrm{mol}$ per molecule, or $\sim 10 \%$ of the energy of an optimal $\mathrm{H}-$ bond. For a given bis-urea molecule, the H-bond energy difference between the three morphologies is even smaller. The largest difference, $0.7 \mathrm{kcal} / \mathrm{mol}$, is found for bis-urea $\mathbf{2}$, between the helical structure on one hand, and the zigzag and straight structures on the other hand. In all cases the H-bond network persists throughout the MD simulations.

Concerning $\pi-\pi$ stacking interactions, the situation is very dependent on the morphology. In the helical morphology adjacent aromatic rings are placed in close contact, with a distance down to $3.5 \AA$, and are therefore stabilized by $\pi-\pi$ stacking interactions. This is also the case for the zigzag morphology, but only within triads (see Supporting Information). In contrast, in the straight morphology, adjacent bis-ureas position their aromatic groups on opposite sides of the hydrogen bonded urea network (Figure 2 ), so that $\pi-\pi$ stacking is impossible.

The influence of $\pi-\pi$ interactions on the stability of the assemblies is expected to be most visible for the system where there is minimal influence of the organization of side groups, which could overcome any change of $\pi-\pi$ interactions from one morphology to the next. It is thus in bis-urea $\mathbf{4}$, the system with the smallest side group, that we will search any energetic effect due to change of $\pi-\pi$ interactions between assemblies. Figure 6 shows the potential energy of the assemblies, including all intra- and intermolecular interactions. The stability decreases from continuous $\pi$ stacking (helical), to partial $\pi$ stacking (zigzag), to absent $\pi$ stacking (straight), thus suggesting that $\pi-\pi$ interactions do stabilize the assemblies that have them. But these energy differences are indeed dwarfed by the interactions between side groups, when their size is larger. See in Figure 6, bis-urea 1, where the energy difference between the morphologies is too small to hierarchize them; or bis-ureas $\mathbf{2}$ and $\mathbf{3}$, where the influence of the side group is clearly dominant. 

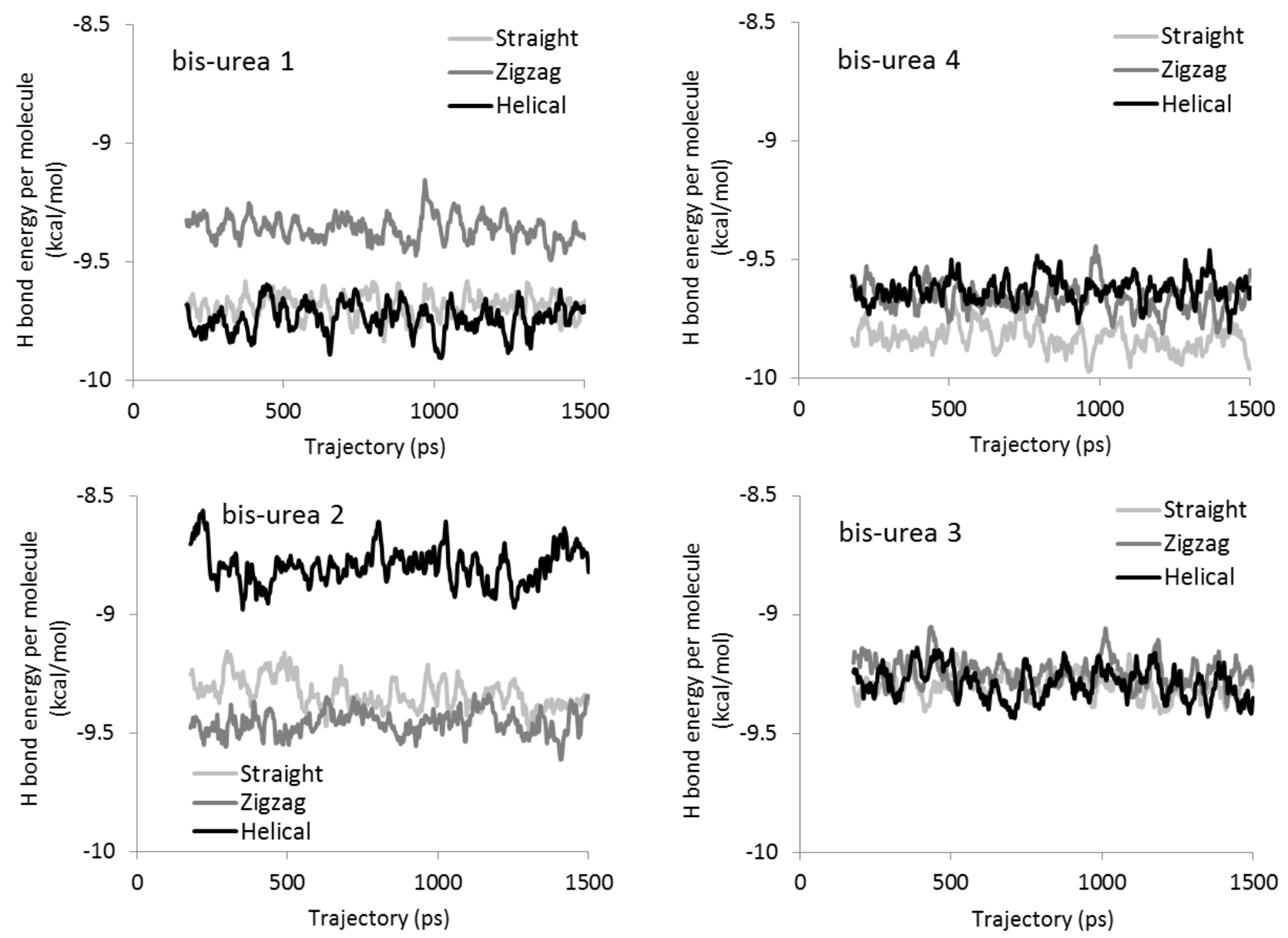

Figure 5 : Plots of the H-bond energy per molecule during the MD simulations for the helical, zigzag, and straight morphologies of the four urea-based systems. The energy values are block averages over ten successive MD structures. See Table S5 for averages and standard deviations. 

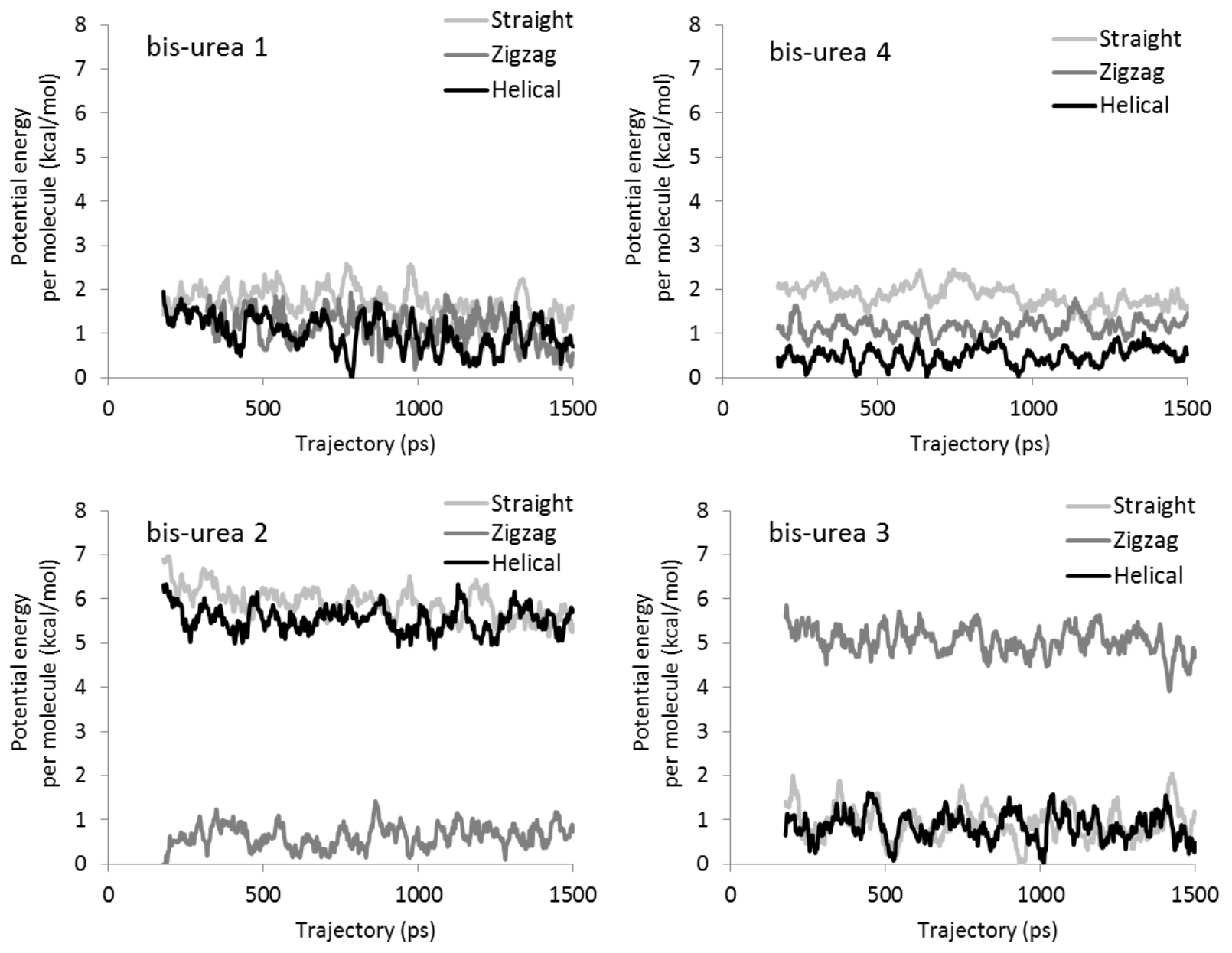

Fig.

per molecule during the MD simulations for the helical, zigzag, and straight morphologies of the four systems. The energy values are block averages over ten successive MD structures, and the zero of the energy scale corresponds to the lowest-energy block average found during the MD runs on the three morphologies. See Table S6 for averages and standard deviations.

In the rest of the discussion, we will thus focus on the influence of the side groups. From that point of view, a distinction can be made between bis-ureas $\mathbf{1}$ and $\mathbf{4}$ on one hand, and $\mathbf{2}$ and $\mathbf{3}$ on the other hand. For 1 and 4, the range of total potential energy within which the morphologies lie is merely 0.7 and 1.4 $\mathrm{kcal} / \mathrm{mol}$, respectively (Figure 6 and Table S6). Since the energy differences are quite small between the three morphologies, it is likely that the substituents have room to organize and do not exert strong steric constraints. Despite small energy differences between them, each morphology is however quite stable, i.e. they do not convert into each other, nor evolve into a mixing of morphologies during the MD 
simulations. It is a feature also shared by the other bis-urea systems studied here. Similarly, the population distributions of $\varphi_{1}, \varphi_{2}$ are narrow for all systems (Figure 7), meaning that the conformation of the bis-urea cores is maintained during the MD simulations, and they are similar whatever the side groups, and whatever the environment (for the simulated single filaments or the two experimental crystalline filaments). These observations reflect the steepness of the potential energy valley in which these morphologies lie once the filament is formed. The stability of the assemblies is attributed to cooperative effects, in particular to cooperative H-bonds, between neighboring molecules.

Based on these simulations, the three morphologies could exist for bis-ureas $\mathbf{1}$ and $\mathbf{4}$, and the actual morphology adopted by these systems in a given environment most probably results from a subtle balance of intra-filament and extra-filament interactions. For instance, experimentally it was found that filaments of enantiomerically pure bis-urea 1 in solution show no CD signal, ${ }^{28}$ suggesting that the morphology is non-helical, i.e. straight or zigzag. 

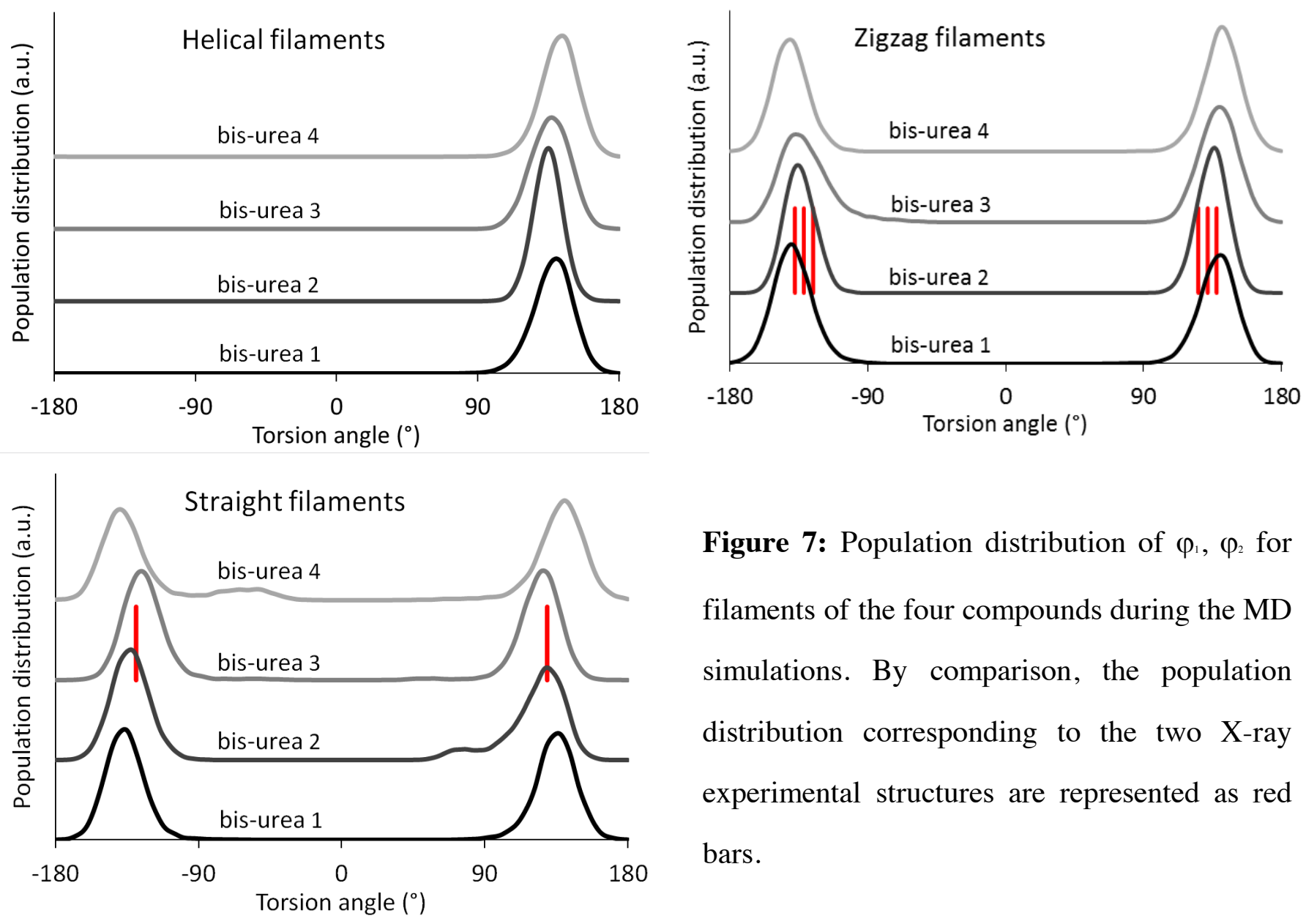

Figure 7: Population distribution of $\varphi_{1}, \varphi_{2}$ for filaments of the four compounds during the MD simulations. By comparison, the population distribution corresponding to the two X-ray experimental structures are represented as red bars.

For bis-ureas $\mathbf{2}$ and $\mathbf{3}$, the difference of potential energy between the morphologies is about 5 and 4 $\mathrm{kcal} / \mathrm{mol}$, respectively (Figure 6 and Table S6). This is a large value, which is also much larger than the differences in the H-bond energy (below $1 \mathrm{kcal} / \mathrm{mol}$ ) between the morphologies. This result indicates 
that the structure of the substituents has here a strong influence on the morphology adopted by the filament, and that some morphologies are clearly destabilized by steric interactions.

As bis-ureas $\mathbf{1}$ and $\mathbf{2}$ are structural isomers, the differences between the two systems in terms of stability of the three morphologies are especially noteworthy. For bis-urea $\mathbf{2}$, the zigzag morphology is much more stable than the straight and helical morphologies, by about $5 \mathrm{kcal} / \mathrm{mol}$. The zigzag morphology is also the morphology that is found experimentally in crystals. Thus, the intra-filament interactions are here highly selective, as they give rise to the same organization both in the presence of inter-filament interactions (in the experiment) and in the absence of such interactions (in the MD simulations). The presence of a quaternary carbon directly attached to the urea moieties for bis-urea $\mathbf{2}$, instead of a secondary carbon for bis-urea 1, probably limits the range of orientations of the molecules for which steric hindrance is small and simultaneously the hydrogen bond network is strong, which leads to the "selection" of a single morphology (i.e., zigzag). The same reasons can be invoked for explaining the widening of the energy differences between the morphologies of bis-urea 3 when compared to those of bis-urea $\mathbf{1}$. In bis-urea $\mathbf{3}$, the second atom of the side group is a quaternary carbon, instead of a tertiary carbon in bis-urea 1. For bis-urea $\mathbf{3}$, the simulated straight and helical morphologies have similar characteristics in terms of potential energy and hydrogen bond energy. The fact that it is the straight morphology that is found experimentally in crystals indicates that other interactions not present in single filaments are significant enough to favor one of the two morphologies in the solid state. In that selection, inter-filament interactions likely play a significant role. In solution, however, interactions with the solvent are likely the dominant interactions tilting the energy balance towards one of the two morphologies. Globally, these results show the importance of the branching and size of the side group in determining the actual morphology of the filament.

\section{Conclusion}

The careful exploration of the energy landscape of bis-urea supramolecular polymers has allowed to identify three distinct self-assembled structures of similar stabilities, two of which are also found in Xray crystal data. In fact, a slight change in the molecular structure can favor a particular structure over the others. Detailed analysis shows that hydrogen bonds stabilize all three structures to a similar extent, 
whatever the side groups bulkiness. However, the total potential energy is strongly affected by the nature of the side groups. Therefore, interactions among the lateral substituents, and with the filament environment, appear to be the decisive factor in the competition between the possible self-assembled structures. This study constitutes a clear reminder that the conformation of a supramolecular polymer is a sensitive function of the molecular structure and may significantly differ from the solid-state conformation of a model compound.

ACKNOWLEDGMENT. Research in Mons is supported by the Science Policy Office of the Belgian Federal Government (IAP 7/05), the OPTI2MAT Excellence Program of Région Wallonne, and FNRSFRFC. The Paris-Mons collaboration takes place in the frame of the ANR-12-BS08-0019-01 project BalanceSupra. Goulnara Garipova and Mihaela Roman are acknowledged for providing compounds 2 and 3. M. L. thanks SERC (Swedish e-Science Research Center) for funding.

SUPPORTING INFORMATION AVAILABLE. Synthesis, crystal structure data and additional simulation results. This information is available free of charge via the Internet at http://pubs.acs.org

\section{REFERENCES}

(1) Brunsveld, L.; Folmer, B. J. B.; Meijer, E. W.; Sijbesma, R. P. Supramolecular Polymers. Chem. Rev. 2001, 101, 4071-4097.

(2) Supramolecular Polymers; Ciferri, A., Ed.; Marcel Dekker, Inc.: New York, 2005.

(3) Bouteiller, L. Assembly via Hydrogen Bonds of Low Molar Mass Compounds into Supramolecular Polymers. Adv. Polym. Sci. 2007, 207, 79-112.

(4) De Greef, T. F. A.; Smulders, M. M. J.; Wolffs, M.; Schenning, A. P. H. J.; Sijbesma, R. P.; Meijer, E. W. Supramolecular Polymerization. Chem. Rev. 2009, 109, 5687-5754.

(5) Palmans, A. R. A.; Meijer, E. W. Amplification of Chirality in Dynamic Supramolecular Aggregates. Angew. Chem. Int. Ed. 2007, 46, 8948-8968. 
(6) Roman, M.; Cannizzo, C.; Pinault, T.; Isare, B.; Andrioletti, B.; van der Schoot, P.; Bouteiller,

L. Supramolecular Balance: Using Cooperativity to Amplify Weak Interactions. J. Am. Chem. Soc. 2010, $132,16818-16824$.

(7) Bouteiller, L.; van der Schoot, P. Probing Intermolecular Interactions in Self-Assembled Nanotubes. J. Am. Chem. Soc. 2012, 134, 1363-1366.

(8) Danila, I.; Riobe, F.; Piron, F.; Puigmarti-Luis, J.; Wallis, J. D.; Linares, M.; Agren, H.; Beljonne, D.; Amabilino, D. B.; Avarvari, N. Hierarchical Chiral Expression from the Nano- to Mesoscale in Synthetic Supramolecular Helical Fibers of a Nonamphiphilic C3-Symmetrical $\pi$ Functional Molecule. J. Am. Chem. Soc. 2011, 133, 8344-8353.

(9) George, S. J.; de Bruijn, R.; Tomovic, Z.; Van Averbeke, B.; Beljonne, D.; Lazzaroni, R.; Schenning, A. P. H. J.; Meijer, E. W. Asymmetric Noncovalent Synthesis of Self-Assembled OneDimensional Stacks by a Chiral Supramolecular Auxiliary Approach J. Am. Chem. Soc. 2012, 134, 17789-17796.

(10) Nakano, Y.; Hirose, T.; Stals, P. J. M.; Meijer, E. W.; Palmans, A. R. A. Conformational Analysis of Supramolecular Polymerization Processes of Disc-like Molecules. Chem. Sci. 2012, 3, 148155.

(11) Hanabusa, K; Koto, C.; Kimura, M.; Shirai, H.; Kakehi, A. Remarkable Viscoelasticity of Organic Solvents Containing Trialkyl-1,3,5-benzenetricarboxamides and their Intermolecular Hydrogen Bonding. Chem. Lett. 1997, 429-430.

(12) Brunsveld, L.; Schenning, A. P. H. J.; Broeren, M. A. C.; Janssen, H. M.; Vekemans, J. A. J. M.; Meijer, E. W. Chiral Amplification in Columns of Self-Assembled $N, N^{\prime}, N^{\prime \prime}-\operatorname{Tris}((S)-3,7-$ dimethyloctyl)benzene-1,3,5-tricarboxamide in Dilute Solution. Chem. Lett. 2000, 29, 292-293.

(13) Araki, K.; Takasawa, R.; Yoshikawa, I. Design, Fabrication, and Properties of Macroscale Supramolecular Fibers Consisted of Fully Hydrogen-bonded Pseudo-polymer Chains. Chem. Commun. 2001, 1826-1827. 
(14) Koevoets, R. A.; Versteegen, R. M.; Kooijman, H.; Spek, A. L.; Sijbesma, R. P.; Meijer, E. W. Molecular Recognition in a Thermoplastic Elastomer. J. Am. Chem. Soc. 2005, 127, 2999-3003.

(15) Stoncius, S.; Orentas, E.; Butkus, E.; Ohrström, L.; Wendt, O. F.; Wärnmark, K. An Approach to Helical Tubular Self-Aggregation Using C2-Symmetric Self-Complementary Hydrogen-Bonding Cavity Molecules. J. Am. Chem. Soc. 2006, 128, 8272-8285.

(16) Bouteiller, L.; Colombani, O.; Lortie, F.; Terech, P. Thickness Transition of a Rigid Supramolecular Polymer. J. Am. Chem. Soc. 2005, 127, 8893-8898.

(17) Bellot, M.; Bouteiller, L. Thermodynamic Description of Bis-Urea Self-Assembly: Competition Between Two Supramolecular Polymers. Langmuir 2008, 24, 14176-14182.

(18) Shikata, T.; Nishida, T.; Isare, B.; Linares, M.; Lazzaroni, R.; Bouteiller L. Structure and Dynamics of a Bisurea Based Supramolecular Polymer in n-Dodecane J. Phys. Chem. B 2008, 112, 8459-8465.

(19) Simic, V.; Bouteiller, L.; Jalabert, M. Highly Cooperative Formation of Bis-Urea Based Supramolecular Polymers. J. Am. Chem. Soc. 2003, 125, 13148-13154.

(20) Arnaud, A.; Bouteiller, L. Isothermal Titration Calorimetry of Supramolecular Polymers. Langmuir 2004, 20, 6858-6863.

(21) Vonau, F.; Suhr, D.; Aubel, D.; Bouteiller, L.; Reiter, G.; Simon, L. Evolution of Multilevel Order in Supramolecular Assemblies. Phys. Rev. Lett. 2005, 94, 066103.

(22) Vonau, F.; Aubel, D.; Bouteiller, L.; Reiter, G.; Simon, L. Cooperative Rearrangements Leading to Long Range Order in Monolayers of Supramolecular Polymers. Phys. Rev. Lett. 2007, 99, 086103.

(23) Vonau, F.; Linares, M.; Isare, B.; Aubel, D.; Habar, M.; Bouteiller, L.; Reiter, G.; Geskin, V.; Zerbetto, F.; Lazzaroni, R. et al. Branched Substituents Generate Improved Supramolecular Ordering in Physisorbed Molecular Assemblies. J. Phys. Chem. C 2009, 113, 4955-4959. 
(24) Mayo, S. L.; Olafson, B. D.; Goddard III, W. A. DREIDING: a Generic Force Field for Molecular Simulations. J. Phys. Chem. 1990, 94, 8897-8909.

(25) For the helical structure (see below), we also considered an assembly where the stacked molecules have overlapping, instead of alternating, orientation of the methyl group on the toluene ring. This orientation for the methyl groups was thought to be more appropriate for helical structures, and for the system for which we made the modification, bis-urea $\mathbf{1}$, the stability indeed appears to be slightly improved, but not significantly.

(26) Nosé, S. A Molecular Dynamics Method for Simulations in the Canonical Ensemble. Mol. Phys. 1984, $52,255-268$.

(27) Verlet, L. Computer Experiments on Classical fluids. I. Thermodynamical Properties of Lennard-Jones Molecules. Phys. Rev. 1967, 159, 98-103.

(28) Isare, B.; Linares, M.; Zargarian, L.; Fermandjian, S.; Miura, M.; Motohashi, S.; Vanthuyne, N.; Lazzaroni, R.; Bouteiller, L. Chirality in Dynamic Supramolecular Nanotubes Induced by a Chiral Solvent. Chem. Eur. J. 2010, 16, 173-177. 